\title{
8x14Gb/s Si Ring WDM Modulator Array with Integrated Tungsten Heaters and Ge Monitor Photodetectors
}

\author{
M. Pantouvaki ${ }^{1}$, P. Verheyen ${ }^{1}$, G. Lepage ${ }^{1}$, J. De Coster ${ }^{1}$, H. Yư ${ }^{2}$, P. De Heyn ${ }^{2}$, A. Masood ${ }^{2}$, W. Bogaerts ${ }^{2}$, P. \\ Absil ${ }^{1}$ and J. Van Campenhout ${ }^{1}$ \\ ${ }^{1}$ Imec, Kapeldreef 75, Leuven B-3001, Belgium \\ ${ }^{2}$ Photonics Research Group, Dept. of Information Technology, Ghent University - imec, St. Pietersnieuwstraat 41, 9000 Ghent, Belgium \\ Author e-mail address: pantouvm@imec.be
}

\begin{abstract}
An 8x14Gb/s wavelength-division multiplexed Si ring modulator array is presented with uniform channel performance. Tungsten heaters and Ge monitor photodetectors at the ring modulator drop ports are co-integrated to track and control the modulation quality.

OCIS codes: (200.4650) Optical interconnects; (230.4110) Modulators; (230.0250) Optoelectronics
\end{abstract}

\section{Introduction}

Silicon carrier depletion ring modulators are attractive for optical interconnects because they require low operating energy and they can support high bit-rates, while occupying small footprint [1,2]. They have therefore the potential to enable significant bandwidths in wavelength division multiplexed (WDM) configurations, at reasonable cost. However, due to their resonant nature, ring modulators suffer from narrow operating bandwidth. Resonance wavelength tunability is necessary to maintain error-free modulation during temperature fluctuations and to compensate for fabrication variability. Doped silicon or metal heaters have been demonstrated on ring modulators to achieve wavelength tuning [1-3]. In [3] we presented tungsten (W) heaters integrated in a standard CMOS BEOL flow, for the thermal control of photonic waveguides. In [4], we demonstrated the co-integration of ring modulators with Ge photodetectors (PDs) for monitoring resonance shifts. In this paper we present an 8-channel WDM transmitter comprised of ring modulators with drop ports, W-heaters and a Ge photodetector at each drop port. The metal heaters enable wavelength tuning of the ring modulator, while the Ge photodetectors can be used in a feedback loop as the monitoring device to trigger resonance tuning when needed. We show $0.19 \mathrm{~nm} / \mathrm{mW}$ heater tuning efficiency on all channels, $3 \mathrm{~dB}$ bandwidths of $8.5 \mathrm{GHz}$ at $0 \mathrm{~V}$ for the ring modulators and $3 \mathrm{~dB}$ bandwidths of $18 \mathrm{GHz}$ at $-1 \mathrm{~V}$ for the Ge photodetectors. Large-signal modulation with open eye diagrams at $14 \mathrm{~Gb} / \mathrm{s}$ is also demonstrated using a $1.5 \mathrm{~V}$ peak-to-peak $\left(\mathrm{V}_{\mathrm{pp}}\right) \mathrm{RF}$ drive signal corresponding to only $7 \mathrm{fJ}$ energy/bit per ring modulator, excluding heater power consumption. Operation at fixed wavelength is studied when the heater power is varying from $2.5-5 \mathrm{~mW}$, and it is found that optimum eye diagrams corresponding to minimum transmitter penalty (TP) can be correlated to Ge PD photocurrent.

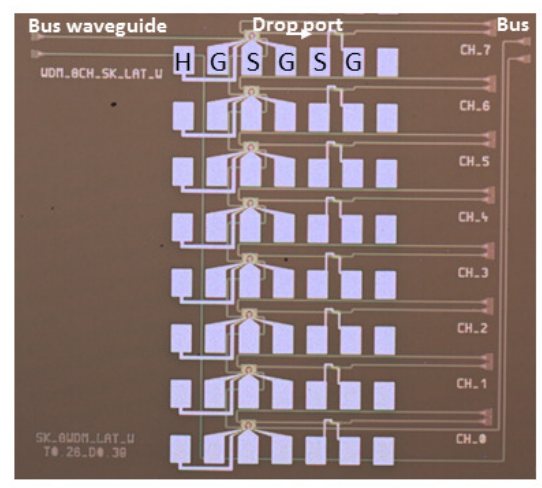

(a)

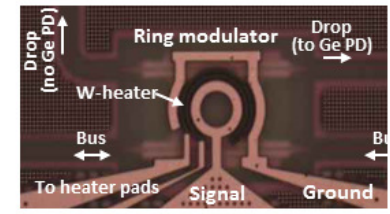

(b)

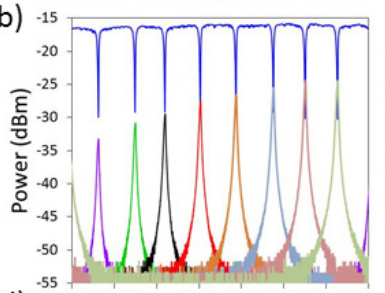

(d) ${ }^{-5548} 1550155215541556155815601562$ wavelength $(\mathrm{nm})$ (c)

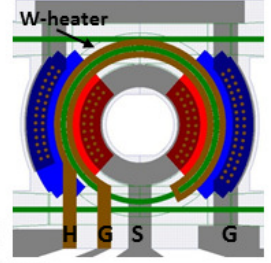

Fig. 1. Top view of (a) the 8 channel WDM transmitter with integrated W-heaters and Ge photodiodes, and (b) focus on the ring modulator. (c) Detail of the modulator design. (d) Transmission spectra of 8 cascaded ring modulators with drop ports.

\section{Device description and DC performance}

Fig. 1(a) shows a top view of the fabricated array of 8 ring modulators with drop ports, W-heaters and Ge photodetectors at the drop port. A zoom-in of the ring modulators and a detail from the ring design are also shown in Fig. 1(b) and Fig. 1(c) respectively. In this design, when light is input at the left side of the bus waveguide of the 
cascaded rings, the drop ports can be characterized with optical transmission measurements, while when light is input at the right side of the bus the response of the Ge photodetectors to the light in the drop ports can be measured
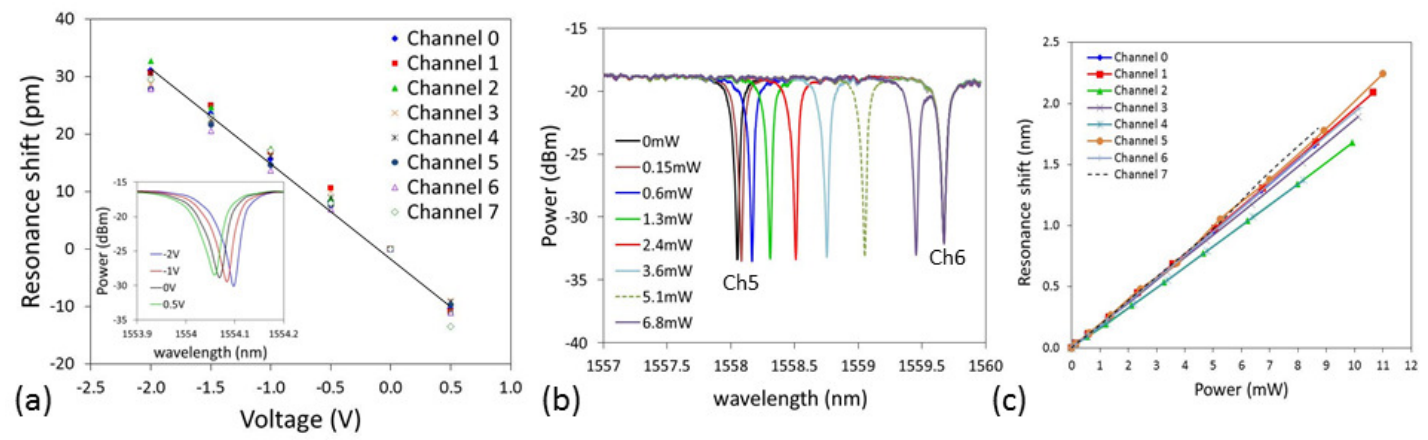

Fig. 2. (a) Resonance shift versus reverse bias for the 8 ring modulators and example of transmission spectra with applied bias. (b) Wavelength tuning of modulator resonance with applied heater power. (c) Ring resonance shift versus heater power for 8 channels.

The silicon ring waveguides had approximately $7.5 \mu \mathrm{m}$ radius and they were formed by etching $150 \mathrm{~nm}$ out of $220 \mathrm{~nm}$ crystalline silicon on $2 \mu \mathrm{m}$ buried oxide. A p-n diode (doped at $4 \mathrm{e} 18 \mathrm{~cm}^{-3}$ ) was implemented only in about $50 \%$ of the ring circumference, as shown in Fig. 1(c), resulting in $\sim 12 \mathrm{fF}$ ring capacitance. The ring radius was slightly varied from ring to ring in the cascaded configuration along the same waveguide, resulting in an average channel spacing of $1.6 \pm 0.12 \mathrm{~nm}$ along with a free spectral range (FSR) of $\sim 12.8 \mathrm{~nm}$, as shown in Fig. 1(d). A reduction of $\sim 7 \mathrm{~dB}$ in the drop port transmission with increasing propagation distance along the bus waveguide was measured, which is attributed to increasing loss in waveguide transitions from deep-to-shallow etched waveguides. This could be improved in a future optimized design. An example of the ring response with applied DC bias is shown in the inset of Fig. 2(a). The modulation efficiency of all rings was $\sim 16 \mathrm{pm} / \mathrm{V}$, as shown in Fig. 2(a). The Qfactor of the rings at $0 \mathrm{~V}$ bias was about $18500 \pm 400$ for all rings and it was the main limitation of the rings' smallsignal $\left(S_{21}\right)$ frequency response to $f_{3 \mathrm{~dB}}<10.5 \mathrm{GHz}$. By optimizing the design it is expected that the bandwidth can be increased to $20 \mathrm{GHz}$, enabling $28 \mathrm{~Gb} / \mathrm{s}$ operation.

The $\mathrm{W}$-heaters were formed about $1 \mu \mathrm{m}$ on top of the ring modulators, after the $\mathrm{W}$-contact holes and before the $\mathrm{Cu}$ metallization of the metal connections, as described in [3]. Fig. 2(b) shows an example of the resonance shift with applied heater power, while Fig. 2(c) shows the resonance shift with heater power for all 8 channels. The heating efficiency was $190 \pm 15 \mathrm{pm} / \mathrm{mW}$, or about $67.4 \mathrm{~mW}$ for one FSR of a single ring. This translates to approximately $8.5 \mathrm{~mW}$ needed for shift of the resonance within the $\sim 1.6 \mathrm{~nm}$ of the channel spacing. No degradation of the extinction ratio was observed with heater power, as shown in Fig. 2(b). Local substrate undercut is expected to substantially improve the power efficiency of the heaters [3].

The Ge photodetectors were lateral p-i-n diodes, $15 \mu \mathrm{m}$ long, formed by selective Ge epitaxial growth on top of the crystalline silicon and before the contact module. The dark current of these devices was about 100nA at $-1 \mathrm{~V}$ and the responsivity was above $0.55 \mathrm{~A} / \mathrm{W}$ at $1555 \mathrm{~nm}$ for all channels.
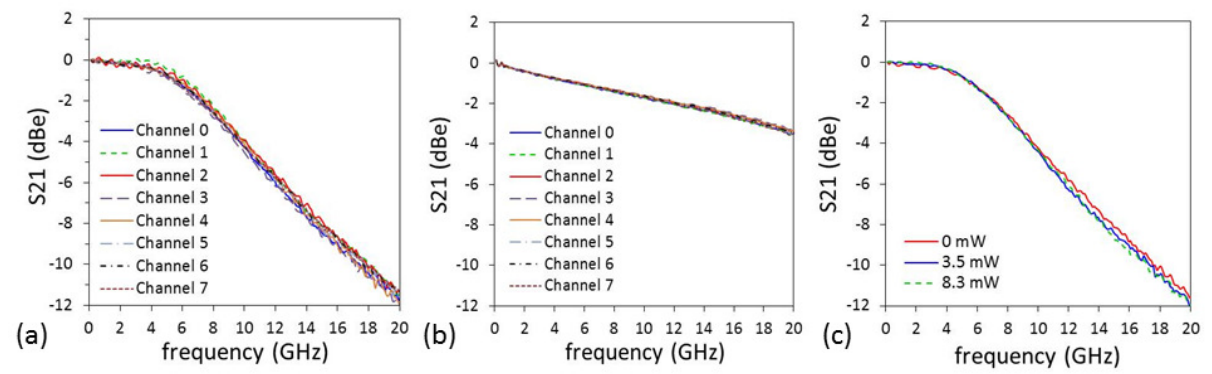

Fig. 3. Frequency response of (a) silicon ring modulators at $0 \mathrm{~V}$, and (b) Ge photodetectors at $-1 \mathrm{~V}$ for all 8 channels. (c) Frequency response of the ring modulator at channel 6 at different $\mathrm{W}$-heater powers.

\section{High-speed characterization}

Small-signal $\mathrm{S}_{21}$ parameter characterization was performed using an Agilent network analyzer at -8dBm RF power. The frequency response of the silicon ring modulators and the Ge photodetectors is shown in Fig. 3(a) and 3(b) respectively. A $3-\mathrm{dB}$ bandwidth of $8.6 \mathrm{GHz}$ was measured for all ring modulators at $0 \mathrm{~V}$. The Ge photodetectors had 
a 3-dB bandwidth of $18 \mathrm{GHz}$ at $-1 \mathrm{~V}$. The effect of heater power on the frequency response of the modulators is shown in Fig. 3(c). No difference in the small-signal performance of the ring modulators was measured for up to $8.3 \mathrm{~mW}$ W-heater power.

The transmitter penalty (TP) can be defined as:

$$
T P(d B)=-10 \log _{10}\left(\frac{P_{1}-P_{0}}{2 P_{i n}}\right)
$$

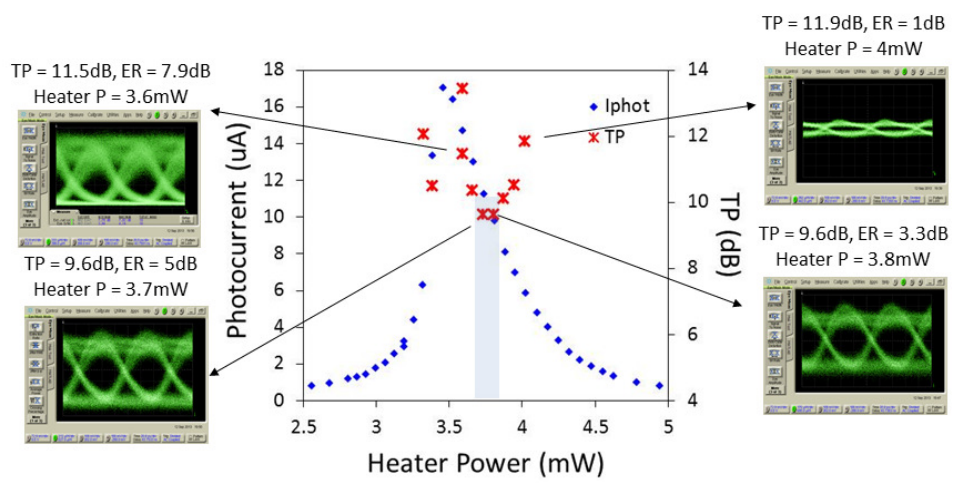

Fig. 4. Dynamic transmitter penalty and Ge photocurrent measured at $14 \mathrm{~Gb} / \mathrm{s}$ on channel 3 at fixed wavelength of $1555.4 \mathrm{~nm}$, with varying power on the $\mathrm{W}$-heater. $14 \mathrm{~Gb} / \mathrm{s}$ eye diagrams measured at different $\mathrm{W}$-heater power are also shown for comparison. Best eye diagrams at this wavelength for channel 3 were obtained at heater power indicated by the shaded area -this best operating point coincides with minimum TP.

The transmitter penalty is a useful figure of merit to determine the best wavelength of operation for low-power ring modulators [4]. Here, we use TP to assess modulation quality at fixed wavelength when the ring resonance is tuned by varying the W-heater power, and we correlate TP to the Ge photocurrent. Fig. 4 shows the Ge photocurrent and the dynamic TP with increasing $\mathrm{W}$-heater power, measured when the ring was operated at $14 \mathrm{~Gb} / \mathrm{s}$, driven by a $1.5 \mathrm{~V}_{\mathrm{pp}}$ signal with a $-1.5 \mathrm{~V}$ reverse bias, and using a $2^{31}-1$ PRBS input signal. The operating wavelength during these measurements was fixed to $1555.4 \mathrm{~nm}$ and the optical power arriving at the modulator array was $-2 \mathrm{dBm}$. It is shown that as the ring resonance is red-shifted with increasing heater power, the photocurrent is also varied, because the ring acts as a filter for the power incident on the Ge photodetector. Maximum photocurrent is therefore measured at heater power when the ring resonance minimum is at $1555.4 \mathrm{~nm}$. Although this results in significant Extinction Ratio $(E R=7.9 \mathrm{~dB})$, this condition coincides also with high insertion loss (IL), which does not result in optimum eye diagram (see top left eye in Fig. 4). Significant noise on the 1-bits is detected at this operating point, when the eye is measured using an EDFA and an optical filter after the modulator. Increasing further the heater power results in optimum combination of ER and IL at $1555.4 \mathrm{~nm}$, or minimum TP, as shown by the bottom left and right eye diagrams of Fig. 4. This corresponds to $\sim 10 \mu \mathrm{A}$ average photocurrent on the Ge photodetector, sufficient for signal monitoring by a feedback control circuit. Further increase of the heater power red-shifts the ring resonance away from the wavelength of $1555.4 \mathrm{~nm}$, increasing again TP and degrading eye quality (top right eye diagram in Fig. 4).

\section{Conclusions}

In summary, an $8 \times 14 \mathrm{~Gb} / \mathrm{s}$ wavelength-division multiplexed Si ring modulator array was presented, co-integrated with Tungsten heaters and Ge monitor photodetectors at the ring modulator drop ports. Uniform channel performance was demonstrated in terms of ring modulation bandwidth and efficiency, heater efficiency and Ge photodetector performance. The co-integration of these various elements will support the future demonstration of actively stabilized, ultra-compact ring-based WDM silicon photonics transmitters.

This work was supported by imec's Core Partner Program. The authors would like to acknowledge contributions from imec's 200mm p-lijn to device fabrication and imec's PDK team to mask tape-out.

\section{References}

[1] X. Zheng et al, "A 33mW 100Gbps CMOS Silicon Photonics WDM Transmitter Using Off-Chip Laser Sources," in Optical Fiber

Communication Conference and the National Fiber Optic Engineers Conference (OFC/NFOEC), (2013), Post-deadline papers, PDP5C.9, pp. 1-3.

[2] G. Li et al, "40Gb/s thermally tunable CMOS ring modulator," in Group IV Photonics (GFP), (2012), pp. 1-3.

[3] A. Masood et al, "Comparison of heater architectures for thermal control of silicon photonic circuits," in Group IV Photonics (GFP), (2013), p. $\mathrm{ThC} 2$.

[4] M. Pantouvaki et al, "20Gb/s Silicon Ring Modulator Co-Integrated with a Ge Monitor Photodetector," in European Conference on Optical Communication (ECOC), (2013), We.3.B.2. 